\title{
Möbius Algorithm for Domain Wall and GapDW Fermions
}

Richard C. Brower*

Boston University

E-mail: brower@bu.edu

Ronald Babich

Boston University

E-mail: rbabich@bu.edu

Kostas Orginos

William \& Mary University

E-mail: kostas@wm.edu

\section{Claudio Rebbi}

Boston University

E-mail: rebbi@bu.edu

\section{David Schaich}

Boston University

E-mail: Schaich@bu.edu

\section{Pavlos Vranas}

Lawrence Livermore National Laboratory

E-mail: vranas2@llnl.gov

The Möbius domain wall action [1] is a generalization of Shamir's action, which gives exactly the same overlap fermion lattice action as the separation $\left(L_{S}\right)$ between the domain walls is taken to infinity. The performance advantages of the algorithm are presented for small ensembles of quenched, full QCD domain wall and Gap domain wall lattices [2]. In particular, it is shown that at the larger lattice spacings relevant to current dynamical simulations Möbius fermions work well together with GapDWF, reducing $L_{s}$ by more than a factor of two. It is noted that there is a precise map between the domain wall and effective overlap action at finite quark mass including finite $L_{s}$ chiral violations so that the Ward-Takahashi identities for the axial and vector currents are exactly equivalent in the two formulations.

The XXVI International Symposium on Lattice Field Theory

July 14 - 19, 2008

Williamsburg, Virginia, USA

\footnotetext{
${ }^{*}$ Speaker.
} 


\section{Introduction}

Domain wall fermions provide an efficient and rigorous implementation of chiral symmetry in lattice field theory at finite lattice spacing. Following the original ideas of Kaplan and Shamir one introduces two 4-d domain walls (or 3 branes) separated by $L_{s}$ lattice sites in a 5 th dimension. The 5-d domain wall action,

$$
S_{D W}=\sum_{x, s}\left[\Psi_{x, s}\left(D_{D W}(m) \Psi\right)_{x, s}+\Phi_{x, s}\left(D_{D W}(1) \Phi\right)_{x, s}\right],
$$

contains 5-d Wilson fermion $\left(\Psi_{x, s}\right)$ and Pauli-Villars pseudo-fermion $\left(\Phi_{x, s}\right)$ fields which enjoy a "kinematical" super symmetry broken only by the boundary conditions on the 3-branes (see Fig. 11). The result is an effective 4-d action with low mass states below the cut-off representing a left(right)

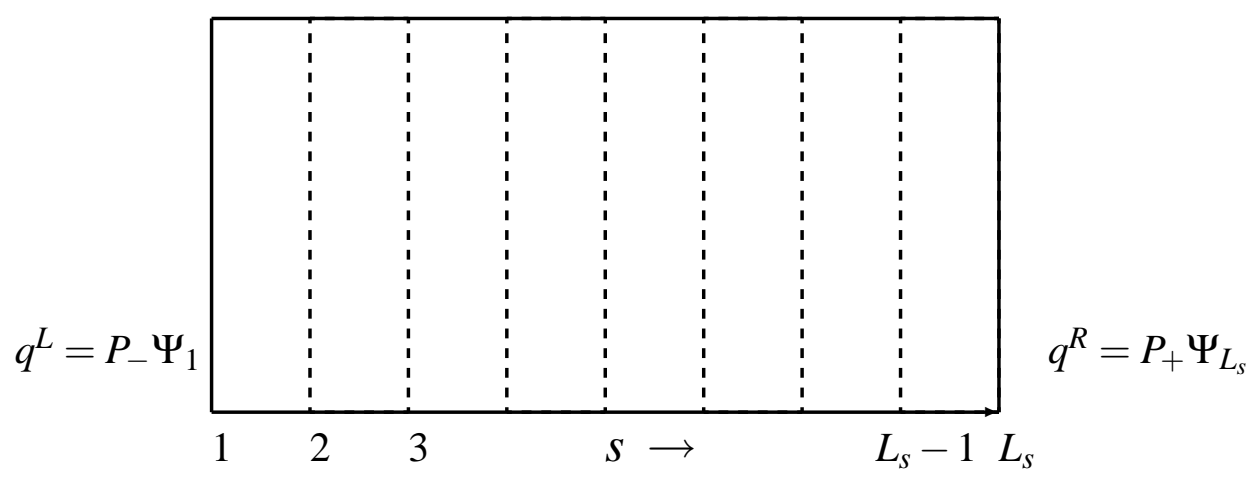

Figure 1: Domain wall convention with left/right chiral mode at $s=1$ and $s=L_{s}$ respectively approximated by 3-branes separated by a distance $a_{5} L_{s}$. The Pauli Villars $(m=1)$ and the zero mass Dirac $(m=0)$ operators obey anti-periodic and Dirichlet boundary conditions respectively.

chiral fermion on each wall at $s=1\left(L_{s}\right)$ respectively in the limit of infinite separation between the walls. After a long and interesting history of competing methods, it is now realized that the effective 4-d theory at $L_{s}=\infty$ is equivalent to one based on Neuberger's overlap operator, with

$$
S_{o v}=\sum_{x y} \psi_{x} D_{o v, x y}(m) \psi_{y} \equiv \psi\left[\frac{1+m}{2}+\frac{1-m}{2} \gamma_{5} \varepsilon[H]\right] \psi
$$

and an appropriate Dirac "Hamiltonian" in the sign function $\varepsilon[H]$. The two actions lead to equivalent matrix elements $D_{o v, x y}^{-1}(m) \equiv\left\langle\psi_{x} \bar{\psi}_{y}\right\rangle_{o v}=\left\langle q_{x} \bar{q}_{y}\right\rangle_{D W}$, where the fields $q, \bar{q}$, shown in Fig. 11, are

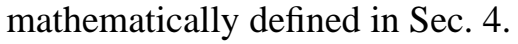

For zero mass quarks, $D_{o v}(0)$ obeys the Ginsparg-Wilson relation,

$$
\gamma_{5} D_{o v}(0)+D_{o v}(0) \gamma_{5}=2 D_{o v}(0) \gamma_{5} D_{o v}(0)
$$

or equivalently the anti-commutator, $\left\{\gamma_{5}, D_{o v}^{-1}(0)\right\}=2 \gamma_{5}$, which guarantees exact chiral symmetry at finite lattice spacing and zero quark mass, $m_{f}=m /(1-m)$. Since all implementations give solutions to the $\mathrm{GW}$ relation, the debate on the virtues of overlap vs. domain wall fermions is essentially algorithmic in nature. For domain wall algorithms, practical considerations demand that 
the $L_{s} \rightarrow \infty$ limit be approximated by modest values: $L_{s}=O(10)$. This is not a trivial requirement. Finite $L_{s}$ causes a residual breaking to the GW relation as measured by the difference operator,

$$
2 \gamma_{5} \Delta_{L_{s}}[H]=\gamma_{5} D_{o v}(0)+D_{o v}(0) \gamma_{5}-2 D_{o v}(0) \gamma_{5} D_{o v}(0)
$$

The conventional criterion for estimating this violation of chiral symmetry is to compare the magnitude of "residual mass",

$$
m_{r e s}=\frac{\operatorname{Tr}\left[D_{o v}^{\dagger-1} \Delta_{L_{s}}[H] D_{o v}^{-1}\right]}{\operatorname{Tr}\left[D_{o v}^{\dagger-1} D_{o v}^{-1}\right]}
$$

relative to the explicit quark mass $m_{f}=m /(1-m)$. As emphasized by Sharpe [3], at current lattices spacings a residual mass $O\left(10^{-3}\right)$ is adequate but some quantities require an order of magnitude smaller residual mass. Moreover recent applications to finite temperature and $\mathscr{N}=1$ SUSY QCD have required much larger values of $L_{s}=O(100)$. Better methods are needed to reduce $m_{\text {res }}$ at reasonable values of $L_{S}$.

Within this framework there still remains a large space of options for the lattice partition function,

$$
Z[U]=\int \mathscr{D} \bar{\psi} \mathscr{D} \psi e^{\beta \operatorname{Tr}\left[U_{P}\right]+S_{\text {improved }}[U]+\bar{\psi} D_{o v}(m)[U] \psi}
$$

by improving the gauge action and/or the approximation to the overlap operator. Here we reexamine the Möbius formulation of the domain wall algorithm [1], verifying that very substantial improvements can be made in the convergence rate to the exact chiral fermion at $L_{s}=\infty$. In addition there is a strong feedback between improved gauge and fermionic algorithms. In particular we point out when the so called "Gap domain wall" modification of the gauge action combines nicely with the Möbius fermion action to give multiplicative improvements - each reducing the residual mass by separate orders of magnitude.

\section{Möbius Recipe}

The simplest kernel for the overlap algorithm is the Wilson Hamiltonian operator, $H=\gamma_{5} D^{\text {Wilson }}\left(M_{5}\right)$, where

$$
D_{x y}^{\text {Wilson }}\left(M_{5}\right)=\left(4+M_{5}\right) \delta_{x, y}-\frac{1}{2}\left[\left(1-\gamma_{\mu}\right) U_{x, x+\mu} \delta_{x+\mu, y}+\left(1+\gamma_{\mu}\right) U_{x, x+\mu}^{\dagger} \delta_{x, y+\mu}\right],
$$

with a negative mass parameter $M_{5} \in[-1,-2]$. However for the domain wall algorithm, the simplest implementation is the Shamir form, $H=\gamma_{5} D^{\text {Shamir }}\left(M_{5}\right)$,

$$
D^{\text {Shamir }}\left(M_{5}\right)=\frac{a_{5} D^{\text {Wilson }}\left(M_{5}\right)}{2+a_{5} D^{\text {Wilson }}\left(M_{5}\right)} .
$$

The Möbius form is a real 3 parameter Möbius transform of the Wilson kernel interpolating between both of these,

$$
D^{\text {Moebius }}\left(M_{5}\right)=\frac{\left(b_{5}+c_{5}\right) D^{\text {Wilson }}\left(M_{5}\right)}{2+\left(b_{5}-c_{5}\right) D^{\text {Wilson }}\left(M_{5}\right)} \equiv \alpha D^{\text {Shamir }}\left(M_{5}\right)
$$


Relative to Shamir's kernel this introduces 1 new "scaling" parameter $\alpha=\left(b_{5}+c_{5}\right) / a_{5}$ at fixed $a_{5}=b_{5}-c_{5}$. Due to the scale invariance of the sign function, $\varepsilon[\alpha \lambda]=\varepsilon[\lambda]$, this does not change the $L_{s}=\infty$ chiral lattice action. Consequently at fixed $a_{5}, M_{5}$ the Möbius rescaling should be regarded as an improved algorithm for the same chiral action, optimized by choosing $\alpha\left(L_{s}\right)$ to minimize $m_{\text {res }}$ at finite $L_{s}$.

Why is this freedom to rescale $H$ desirable? The difficulty with the domain wall approach is that the resultant polar approximation to the sign function,

$$
\varepsilon_{L_{s}}[H]=\frac{(1+H)^{L_{s}}-(1-H)^{L_{s}}}{(1+H)^{L_{s}}+(1-H)^{L_{s}}}=\tanh \left[-\left(L_{s} / 2\right) \log T\right],
$$

is exponentially convergent only for eigenvalues $\lambda$ of $H$ inside the interval: $\log (|\lambda|) \in\left[1 / L_{S}, L_{S}\right]$. So the advantage of rescaling at finite $L_{s}$ is to use this interval more efficiently by shifting the spectrum $\log (\lambda) \rightarrow \log (\alpha)+\log (\lambda)$. Other approaches to improving the polar approximation that have been suggested include explicit projection of a finite set of eigenvalues at small $\lambda$ and/or suppressing the number of small eigenvalues by changing the gauge action. Indeed these may be combined together with Möbius fermions to gain additional advantage as illustrated here by the Möbius rescaling of the GapDW lattices. Next we explain how the domain wall implementation of this rescaling naturally involves the two parameters, $b_{5}, c_{5}$.

The Möbius generalization of Shamir merely requires that the Wilson kernel be included in the 5th dimensional hopping term,

$$
\begin{aligned}
D^{D W}(m)_{s, s^{\prime}} & =D_{-}^{(s)} P_{+} \delta_{s, s^{\prime}+1}+D_{+}^{(s)} \delta_{s, s^{\prime}}+D_{-}^{(s)} P_{-} \delta_{s, s^{\prime}-1} \\
& -m D_{-}^{(1)} P_{+} \delta_{s, 1} \delta_{s^{\prime}, L_{s}}-m D_{-}^{\left(L_{s}\right)} P_{-} \delta_{s, L_{s}} \delta_{s^{\prime}, 1}
\end{aligned}
$$

with $P_{ \pm}=\frac{1}{2}\left(1 \pm \gamma_{5}\right)$ and $D_{+}^{(s)}=b_{5}(s) D^{\text {Wilson }}\left(M_{5}\right)+1, D_{-}^{(s)}=c_{5}(s) D^{\text {Wilson }}\left(M_{5}\right)-1$ with $s, s^{\prime}=$ $1,2, \cdots L_{s}$ or in $L_{s} \times L_{s}$ matrix notation. For the rescaling example discussed we take $b_{5}(s)+c_{5}(s)=$ $\alpha a_{5}, b_{5}(s)-c_{5}(s)=a_{5}$, so the s-dependence for $D_{ \pm}^{(s)}$ can be dropped, however we have included it so that the Möbius class includes other approaches such as the Zolotarev approximation or the variable fields suggested by Bär, Narayanan, Neuberger and Witzel [丹]. In matrix notation:

$$
D^{D W}(m)=\left[\begin{array}{ccccc}
D_{+}^{(1)} & D_{-}^{(1)} P_{-} & 0 & \cdots & -m D_{-}^{(1)} P_{+} \\
D_{-}^{(2)} P_{+} & D_{+}^{(2)} & D_{-}^{(2)} P_{-} & \cdots & 0 \\
0 & D_{-}^{(3)} P_{+} & D_{+}^{(3)} & \cdots & 0 \\
\cdots & \cdots & \cdots & \cdots & \cdots \\
-m D_{-}^{\left(L_{s}\right)} P_{-} & 0 & 0 & \cdots & D_{+}^{\left(L_{s}\right)}
\end{array}\right]
$$

\section{Performance Measures}

Fortunately the new off diagonal Wilson operators in the Möbius domain wall action can be implemented with essentially no additional algorithmic complexity. The first step, suggested in Ref [1], is to replace 5-d red/black preconditioning by a 4-d checker board with no alternation of color along the 5th axis. The new form of the Schur complement solves analytically all interaction in the fifth dimension. The performance of 4-d versus 5-d red/black preconditioning, if anything, 
favors this construction. Then with a simple gather operation for the three spinors in the $\hat{\mu}$ direction, the number of Wilson Dirac application per CG iteration is identical. A full comparison of performance on a range of lattices is impossible in this short talk. So we consider three examples with more to be presented in a future publication.

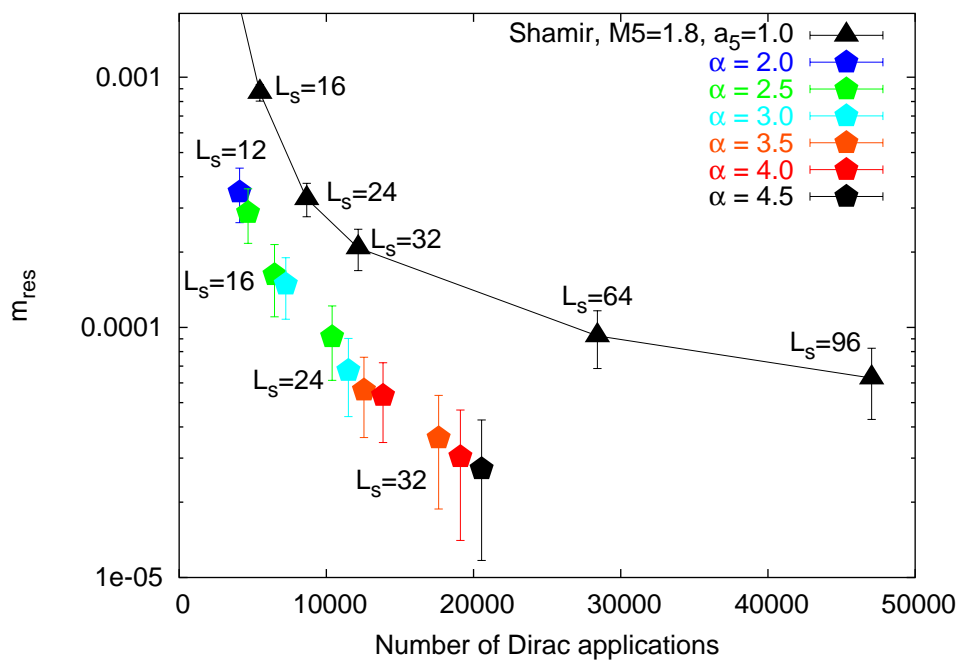

Figure 2: The Möbius algorithm on pure gauge lattices compared with Shamir $(\alpha=1)$.

Quenched Lattices: As demonstrated in the original proposal [1], the Möbius formulation has the potential of an order of magnitude reduction of the explicit chiral symmetry breaking at fixed computational cost. A comparison of the residual masses is given in Fig. 2 on $\beta=6.0$ quenched lattices with $a^{-1} \simeq 2.1 \mathrm{GeV}$. For these lattices the optimal rescaling satisfies the empirical form, $\alpha\left(L_{S}\right) \simeq 1+L_{S} / 8$. Note that for small residual masses the advantage of scaling is huge.

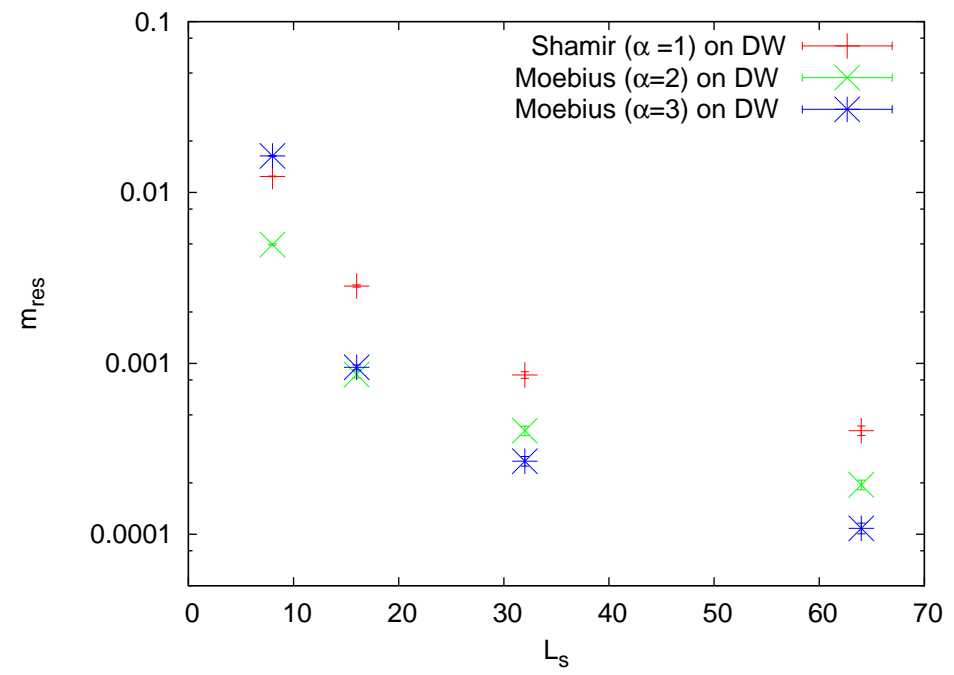

Figure 3: The Möbius algorithm on full QCD domain wall lattices compared to Shamir $(\alpha=1)$.

Domain Wall Lattices: Test were also carried out on full domain wall lattices which generally exhibit worse convergence to small $m_{\text {res }}$. However as an example in Fig. 每 the same comparison is 
made on a set of DWF $N_{f}=2+1$ Iwasaki lattices [阿] at $\beta=2.13$ with $m_{s}=0.04, m_{l} / m_{s}=1 / 4$, $a^{-1} \simeq 1.7 \mathrm{GeV}$. Even without carefully tuning the rescaling parameter the advantage appears to be nearly as dramatic as for the quenched lattices. More thorough studies of this are underway.

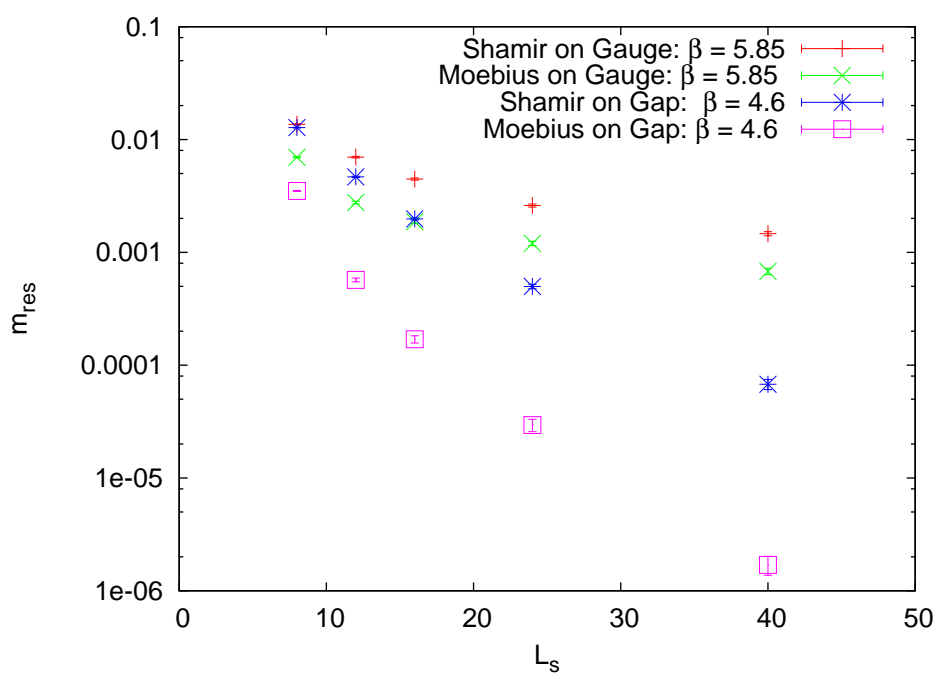

Figure 4: The Möbius algorithm $(\alpha=2)$ on pure gauge and Gap lattices vs Shamir $(\alpha=1)$.

Gapped Lattices: A more radical suggestion to suppress small eigenvalues in $H$ was suggested by Vranas [2] which by adding a pair of Wilson fermions with mass $M_{5}<0$ induces an improvement term,

$$
S_{\text {Gap }}\left[U_{\mu}(x)\right]=\operatorname{Tr}\left[\log \left(D^{\dagger \text { Wilson }}\left(M_{5}\right) D^{\text {Wilson }}\left(M_{5}\right)\right)\right],
$$

for the effective gauge action. To test the locality of this action one should recognize that the bilocal dependence on the gauge field is measured by "axial correlator"

$$
\delta_{U_{\mu}(x)} \delta_{U_{v}(y)} S_{G a p}[U]=\left\langle j_{G a p}^{5 \mu}(x) j_{\text {Gap }}^{5 v}(y)\right\rangle \sim \exp \left[-|x-y| / \xi_{\pi}\right]
$$

whose long distance tail is the would be "pion" propagator for the Gap fermions. Ref. [2] demonstrated that this correlation is $\mathrm{O}(1)$ in lattice units as you approach the continuum limit, roughly the same degree of locality in the overlap action itself. Fig. 1 compares $(\alpha=2)$ vs Shamir $(\alpha=1)$ at $M_{5}=-1.8, m=0.02$ for pure gauge and Gapped lattices both with $a^{-1}=1.4 \mathrm{Gev}$.

\section{Discussion}

Figs. 2 4 point to a general rule that a Möbius scaling transformation of Shamir by a factor of $\alpha>1$ allows one to reduce $L_{s}$ and therefore the cost by a factor of $1 / \alpha$ at fixed $m_{\text {res }}$. This is a natural consequence of the scaling relation, $\Delta_{L_{s}}[\alpha \lambda]=\Delta_{\alpha L_{s}}[\lambda]$, for small eigenvalues, $|\lambda| \leq O\left(1 / L_{s}\right)$. Tuning $\alpha>2$ for large $L_{s}$ gives additional savings. There appears to be an additional computational cost for $\alpha \geq 2$ on the order of $10 \%$ due to increased condition number. Alternatively we note at fixed $L_{s}=O(10)$, the Möbius algorithm can reduce $m_{\text {res }}$ by an order of magnitude or more at fixed cost. The combined improvement in the Möbius algorithm on Gapped lattices is multiplicative so 
here one might contemplate exploratory studies in beyond the standard model strong dynamics with $L_{s}$ in the range of 4 to 8 . For high precision studies $m_{\text {res }}$ can be reduced below $10^{-5}$ for $L_{s}=32$. Both of these are attractive options.

In a subsequent publication more details on the efficiency and formal properties of the Möbius algorithm will be provided. We simply note here that a straight forward general formalism exists that allows all correlators as well as the Ward-Takahashi identities to be expressed independent of the detailed form of the Möbius domain wall action. For example the application of LDU decomposition leads to the basic identity,

$$
\left[\mathscr{P}^{\dagger} \frac{1}{D^{D W}(m)} D^{D W}(1) \mathscr{P}\right]_{s^{\prime} s}=\left[\begin{array}{ccccc}
D_{o v}^{-1}(m) & 0 & 0 & \cdots & 0 \\
(1-m) \Delta_{2}^{R} D_{o v}^{-1}(m) & 1 & 0 & \cdots & 0 \\
(1-m) \Delta_{3}^{R} D_{o v}^{-1}(m) & 0 & 1 & \cdots & 0 \\
\cdots & \cdots & \cdots & \cdots & \cdots \\
(1-m) \Delta_{L_{s}}^{R} D_{o v}^{-1}(m) & 0 & 0 & \cdots & 1
\end{array}\right]_{s^{\prime} s}
$$

where $\mathscr{P}_{s^{\prime}, s}=P_{-} \delta_{s^{\prime}, s}+P_{+} \delta_{s^{\prime}, s+1}$ rotates the Right fermion to the Left wall in Fig. 1. It follows from this that

$$
D_{o v, x y}^{-1}(m) \equiv\left\langle\psi_{x} \bar{\psi}_{y}\right\rangle_{o v}=\left\langle q_{x} \bar{q}_{y}\right\rangle_{D W},
$$

defining the appropriate chiral domain wall fields on the boundary: $q_{x}=[\mathscr{P} \dagger \Psi]_{x, 1}$ and $\bar{q}_{x}=$ $\left[\bar{\Psi} D_{D W}(1) \mathscr{P}\right]_{x, 1}$. All the matrix elements are directly related to the transfer matrix, $T=(1-$ $H) /(1+H)$, through the partial left and right products ${ }^{1}, \Delta_{s}^{L}=T^{-s} /\left(1+T^{-L_{s}}\right)$ and $\Delta_{s+1}^{R}=T^{s-L_{s}} /(1+$ $\left.T^{-L_{s}}\right)$. Together they give the GW chiral breaking operator, $\Delta_{L_{s}}[H]=\Delta_{s}^{L} \Delta_{s+1}^{R}$, defined above in Eq. 1.4. Similar arguments leads to a general map between all overlap and domain wall correlators, with only implicit reference to the Möbius operator $D_{D W}(m)$. From this map, the vector and axial Ward-Takahashi identities must be identical for both overlap and domain wall actions at finite $L_{s}$, lattice spacing and finite volume.

In summary little change in the formalism or software is required to use the Möbius algorithm, while providing a substantial improvement in performance. A very efficient code for the BlueGene has been written by Andrew Polchinski under the SciDAC software project and is readily available at the software links for USQCD: http://usqcd.org.

This work was supported in part by US DOE grant DE-FG02-91ER40676, NSF grant DGE0221680, NSF CCF-0728915, and the Jeffress Memorial Trust grant J-813.

\section{References}

[1] R. C. Brower, H. Neff and K. Orginos, "Möbius fermions: Improved domain wall chiral fermions," Nucl. Phys. Proc. Suppl. 140, 686 (2005)

[2] P. M. Vranas, “Gap domain wall fermions,” Phys. Rev. D 74, 034512 (2006)

[3] S. R. Sharpe, "Future of Chiral Extrapolations with Domain Wall Fermions," arXiv:hep-lat/0706.0218

[4] O. Bär, R. Narayanan, H. Neuberger and O. Witzel, "Domain wall filters," arXiv:hep-lat/0703013.

[5] C Allton et al, "Physical Results from 2+1 Flavor Domain Wall QCD and SU(2) Chiral Perturbation Theory," arXiv:0804.0473

\footnotetext{
${ }^{1}$ For simplicity we have dropped the s-independence of transfer matrix. The general case requires s-ordered product.
} 